\title{
GÊNERO E ESTRESSE EM TRABALHADORES DE UMA UNIVERSIDADE PÚBLICA DO ESTADO DE SÃO PAULO
}

\author{
Maria Elenice Quelho Areias \\ Liliana Andolpho Magalhães Guimarães"
}

\begin{abstract}
RESUMO. Objetivou-se identificar, segundo o gênero, os índices de saúde mental e os fatores psicossociais de risco em trabalhadores de uma universidade pública. A amostra se compôs de 400 trabalhadores - 253 do sexo feminino e 147 do masculino.Utilizou-se o questionário sobre estresse no trabalho intitulado SWS Survey (Self, Work and Social) (Ostermann \& Gutiérrez, 1992), validado para uso no Brasil por Guimarães e Macfadden (1999). Foram usadas estatísticas univariadas, bivariadas e multivariadas. Associações significativas foram encontradas entre: saúde mental e gênero e entre fatores psicossociais de risco e gênero. Participantes do gênero feminino apresentaram mais fatores psicossociais de risco, estresse no trabalho, estresse social e pior saúde mental do que os do gênero masculino $(p \leq 0.05)$, com maior risco de adoecimento físico e/ou mental.
\end{abstract}

Palavras-chave: gênero, estresse, trabalho.

\section{GENDER AND STRESS IN WORKERS OF A PUBLIC UNIVERSITY OF SÃO PAULO STATE}

\begin{abstract}
The aim of this paper was to identify, according to gender, the indexes of Mental Health and the Psychosocial Risk Factors in workers at a state University. A sample of 400 was randomly selected, 253 female and 147 male. They were assessed by means of The Questionnaire SWS Survey (Self, Work and Social) (Ostermann \& Gutiérrez, 1992), validated in Brazil by Guimarães and Macfadden (1999). Univariate, bivariate, multivariate statistics were assessed. Significant associations emerged from Mental Health and Gender, and Psychosocial Risk Factors and Gender. Women showed greater Psychosocial Risk Factors, Work and Social Stress, worst mental health than men $(p \leq 0.05)$, which place them at greater risk for developing physical and/or mental illness.
\end{abstract}

Key words: gender, stress, work.

O interesse pelo estudo sobre as influências do gênero no campo do estresse ocupacional (work stress) é recente. A palavra Gênero não aparece no índice da primeira edição do Handbook of Stress (Goldberger \& Breznitz, 1982), nem em outras publicações importantes nesta área do conhecimento, como Job Stress and Blue Collar Work (Cooper \& Smith, 1985) ou Job Control and Worker Health (Sauter, Hurrell \& Cooper, 1989).
Em 1992, em uma revisão de literatura sobre estresse no trabalho relacionada ao tratamento, contatou-se que a influência do gênero não era mencionada (Holt, 1992). Na maior parte dos estudos em saúde, a questão do gênero tem sido abstraída ou seu efeito tem sido controlado. Este fato parece ocasionar uma escassa visibilidade sobre esta variável, bem como a existência de um corpo de investigações que, na busca da associação entre enfermidade e estresse no trabalho, acaba por reforçar estereótipos.

* Psicóloga do Centro de Saúde da Comunidade (Cecom/Unicamp); membro do Laboratório de Saúde Mental e Trabalho do Departamento de Psicologia Médica e Psiquiatria da Faculdade de Ciências Médicas da Universidade Estadual de Campinas (LSMT/DPMP/FCM/Unicamp).

\# Professora Doutora do Curso de Mestrado em Psicologia da Universidade Católica Dom Bosco (UCDB); Coordenadora do Laboratório de Saúde Mental e Trabalho da UCDB; Coordenadora do Núcleo de Estudos Multidisciplinares em Psicologia (NEMPSI) da UCDB; Professora Doutora convidada do Departamento de Psicologia Médica e Psiquiatria da Faculdade de Ciências Médicas da Universidade Estadual de Campinas (DPMP/FCM/Unicamp); Coordenadora do Laboratório de Saúde Mental e Trabalho do DPMP/FCM/Unicamp. 
Apesar disso, algumas mudanças recentes podem ser observadas.

\section{INFLUÊNCIA DO GÊNERO NAS REAÇÕES DE ESTRESSE E NA SAÚDE MENTAL}

O primeiro estudo especificamente dedicado à influência do gênero em todas as etapas da reação ao estresse foi o Gender and Stress de Barnett, Biener e Baruch (1987). Já em 1992, na segunda edição do Handbook of Stress (Barnett, 1998), alguns capítulos fazem menção à questão do gênero.

Para a compreensão dos efeitos intrínsecos do gênero nas relações estudadas, é preciso levar em consideração as seguintes variáveis relacionadas e associadas ao contexto: o prestígio profissional, o salário, o trabalho em tempo parcial ou tempo integral, o estado civil, a educação, a situação laboral do cônjuge, a carga global de trabalho e a responsabilidade do cuidado de familiares menores ou de idade avançada, e se as mesmas constituem-se em reais variáveis moderadoras e/ou confundidoras ${ }^{1}$, controlando-as adequadamente (Barnett, Marshall, Raudenbush \& Brennan, 1993).

Nos países industrializados, as pesquisas têm-se centrado geralmente nos efeitos do emprego sobre a saúde mental feminina, comparando as mulheres que são exclusivamente donas de casa com as que desenvolvem uma atividade remunerada fora do lar (Ludermir, 2000). Nesta direção, Santana, Loomis e Newman (2001) acrescentam que as mulheres assalariadas relatam menos sintomas psicológicos, ansiedade, sintomas depressivos, angústia psicológica ou sintomas psicossomáticos, quando comparadas a mulheres não assalariadas. $\mathrm{O}$ emprego pode ser benéfico ao seu bem-estar, a depender também da condição de trabalho, da qualidade de sua organização e das percepções subjetivas da pessoa.

Miyata, Tanaka e Tsuji (1997), em estudo sobre o estresse ocupacional, consideraram ser ele resultado de fatores individuais, sociais e laborais. Em pesquisa realizada com 796 pacientes de um ambulatório, os autores estudaram gênero, tipo de emprego/ocupação, tipo de doença e fatores que causam estresse. Foram

\footnotetext{
O uso deste neologismo é necessário. O termo "variável confundidora" refere-se à tradução para o português de "confounding variable", expressão de uso comum e consensual nos estudos que privilegiam o método quantitativo, modelo no qual o presente estudo está inserido. O termo se refere àquelas variáveis que, em não sendo cotejadas e conseqüentemente controladas pelo pesquisador, poderão conduzir a resultados e conclusões incorretas.
}

encontradas diferenças nos resultados envolvendo a categoria gênero. A principal causa de doenças nos pacientes homens $(56 \%)$ foi o estresse ocupacional e, nas mulheres, $(42,4 \%)$ foram os fatores pessoais.

Stuart e Halverson (1997) realizaram um estudo comparativo entre soldados (homens e mulheres) das Forças Armadas dos Estados Unidos, cujas medidas dos sintomas de estresse foram tiradas durante o desdobramento das operações militares no Golfo Pérsico, na Somália, no Kuwait, no Haiti e na Bósnia. Os resultados indicaram que os sintomas de estresse foram significativamente maiores para as mulheres do que para os homens.

Santana, Loomis e Newman (2001), em estudo realizado em áreas urbanas pobres do Nordeste do Brasil, relatam que a sobrecarga de trabalho, que pode ser medida através da dupla jornada ou do tempo total dedicado diariamente ao trabalho, pode ser preditora de altos escores do Questionário de morbidade psiquiátrica do adulto (QMPA) entre mulheres. Os autores observam ainda que mulheres envolvidas somente com o serviço doméstico podem sentir-se frustradas, porque em geral têm habilidades melhores que as ordinariamente requeridas para este tipo de trabalho, o qual normalmente é executado em isolamento e conduz a sentimentos de solidão, como também baixa condição de prestígio e dependência financeira.

Artazcoz, Borrel e Benach (2001) observam que, na Espanha e em outros países, são necessárias mudanças como o compartilhamento de responsabilidades domésticas - bem como políticas públicas ativas para facilitar as tarefas familiares, com vista a reduzir as desigualdades de gênero na saúde, atribuíveis à distribuição desigual das demandas familiares.

Os papéis relacionados ao trabalho e à família têm diferentes significados para homens e mulheres, dados os múltiplos papéis desempenhados por estas últimas. Essas diferenças podem ser parcialmente responsáveis por seus efeitos serem menos benéficos à saúde mental das mulheres quando comparadas aos homens. Acrescenta-se o fato de haver diferenças entre homens e mulheres quanto à natureza e extensão dos conflitos entre trabalho e cuidado dos filhos, das atribuições de responsabilidade aos problemas conjugais, dos sentimentos de culpa e das auto-avaliações como pais e cônjuges (Simon, 1995).

Em pesquisa realizada por Appelberg, Romanov, Heikkila, Honkasalo e Koshenvuo (1996), o conflito interpessoal no trabalho foi considerado preditor de incapacidade para o trabalho somente entre mulheres. Esse risco foi restrito às mulheres que relataram conflitos conjugais simultaneamente. Numa análise 
mais extensa, a insatisfação com a vida em geral foi considerada um fator de risco significativo entre ambos os gêneros, mas o trabalho monótono, o neuroticismo e o estresse vivido nas atividades diárias eram fatores de risco somente para os homens.

À medida que um número crescente de mulheres é incorporado à população ativa e ascende a postos de trabalho tradicionalmente desempenhados pelos homens, aumentam as possibilidades e a necessidade de analisar a influência do gênero na relação entre doença e estresse no trabalho (Barnett, Marshall, Raudenbush \& Brennan, 1993).

É preciso considerar que estudos com amostras compostas de mulheres com ocupações de qualificação mais baixa (como faxineiras, babás, empregadas domésticas, entre outras) tendem a produzir resultados que não se prestam a analisar os efeitos do trabalho na saúde mental, segundo o gênero feminino em geral.

Faz-se necessária a realização de pesquisas com amostras compostas por homens e mulheres pertencentes a estratos ocupacionais similares, para que a questão das diferenças de gênero no estresse ocupacionalpossa ser confirmada ou não, como no presente estudo, tentando-se evitar as falhas mais comuns encontradas em pesquisas sobre o tema.

Para tanto, utilizou-se o instrumento $\mathrm{SWS}^{\odot}$ Survey -(Ostermann, 1989; Ostermann \& Gutiérrez, 1992; Ostermann, Gutiérrez, Contreras \& Atenco, 1995; Areias, 1999), cuja adaptação e validação para o Brasil foram feitas por Guimarães e MacFadden (1999), com base no Modelo de Estresse/Apoio e Saúde Mental, proposto por Ostermann (1989) (Ver Figura 1). O pressuposto teórico do instrumento postula que a saúde mental e os fatores psicossociais de risco são determinados tanto por fatores de apoio quanto por estressores nas dimensões pessoal (self), social e de trabalho. Cada uma dessas dimensões pode contribuir de maneira negativa (como estressor) ou de maneira positiva (como apoio) nas condições gerais da vida.

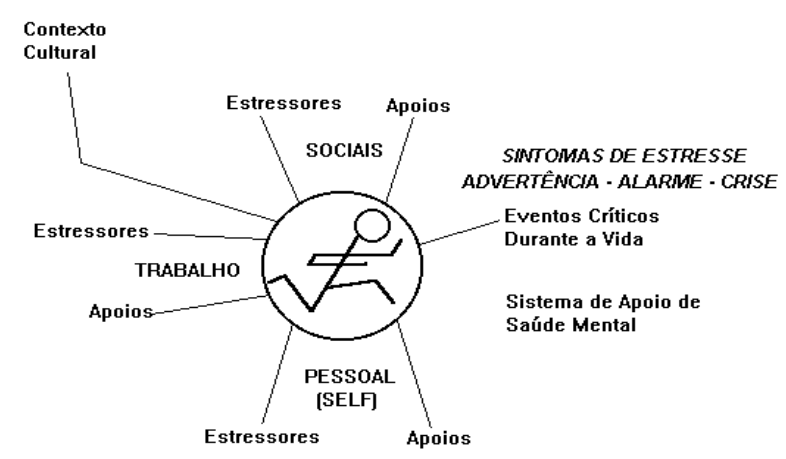

Figura 1. Modelo de Estresse e Apoio e Saúde Mental (Ostermann, 1989).
Embora existam vários instrumentos disponíveis para a avaliação do estresse/saúde mental no trabalho, optou-se pelo uso do SWS, por ser o primeiro instrumento na área a contemplar as variações culturais; estudar cada uma das dimensões (trabalho, pessoal e social) e a sua influência tanto negativa (como estresse) quanto positiva (como apoio) nas condições gerais da vida; por destacar que os fatores de apoio podem anular os efeitos danosos do estresse, permitindo a identificação em separado das variáveis relacionadas às três dimensões pesquisadas (trabalho, social e pessoal) para diferenciação diagnóstica, como podem também possuir uma validação criteriosa para o Brasil (alfa total=0,910, com variação entre 0,901 e 0,976).

O objetivo desse trabalho foi: comparar, entre gêneros, os fatores psicossociais de risco e a saúde mental e os estressores e fatores de apoio nas dimensões pessoal, do trabalho e social.

\section{MÉTODO}

Realizou-se um estudo comparativo, no qual se buscou identificar os índices de saúde mental, os fatores psicossociais de risco, os estressores e fatores de apoio (nas dimensões social, pessoal e trabalho), segundo o gênero. A aplicação do questionário de estresse ocupacional SWS Survey (Self, Work and Social) deu-se em uma única etapa, ao longo dos meses de agosto e setembro de 1998.

Foram cumpridos os princípios éticos contidos na Declaração de Helsinque (1996), da World Medical Association e do Ministério da Saúde do Brasil, para pesquisas envolvendo seres humanos.

\section{Amostra}

Foram estudados trabalhadores de diferentes unidades de um campus universitário estadual situado na cidade de Campinas (SP). De uma população de 8027 pessoas à época do estudo, tomou-se uma amostra aleatória e equiprovável de 400 servidores, correspondendo a 5\% dos homens e 5\% das mulheres do total da população. Excluíram-se os servidores contratados por convênios, bolsistas, inativos e docentes.

\section{Instrumento}

Utilizou-se o questionário SWS (Self, Work and Social), criado por Ostermann e Gutièrrez (1992), com validação brasileira feita por Guimarães e MacFadden (1999). 
Este questionáriofoi aplicado em sua forma completa, a qual é composta de 200 questões, sendo 25 para cada uma das 8 escalas (que representam 8 fatores), descritas a seguir.

1. Fatores psicossociais de risco (FPR): incluem sintomas clássicos e relatos de doenças físicas associadas à instabilidade emocional, diminuição do tônus emocional e comportamento socialmente inadequado, inapropriado ou prejudicial;

2. Saúde mental (SM): compreende os comportamentos de um indivíduo otimista, positivo, realizado, equânime, com auto-estima, boa saúde e uma atitude e comportamento adequados com as pessoas e o meio;

3. Estresse social (ES): se refere asituações que ocorrem fora do âmbito do trabalho e incluem condições de vida caóticas, perigosas ou insalubres, relações conflitivas na dinâmica familiar, com amigos e vizinhos, e responsabilidades que envolvam tensões;

4. Apoio social (AS): se relaciona ao apoio de amigos e parentes,permitindo conforto físico e psicológico à pessoa que vivencia uma situação estressante; compreende situações ocorridas fora do âmbito do trabalho; inclui relações compatíveis, úteis e satisfatórias e condições de vida confortáveis e protegidas;

5. Estresse no trabalho (ET): se refere aos fatores de estresse no ambiente de trabalho que são devidos à natureza do trabalho e suas condições, a conflitos interpessoais entre o trabalho e as obrigações pessoais e às necessidades pessoais de autorealização;

6. Apoio no trabalho (AT): inclui condições de trabalho que propiciam plenamente o desenvolvimento da potencialidade da pessoal, trabalho coma alta significação, boas relações interpessoais e de apoio em geral, condições de trabalho confortáveis;

7. Estresse pessoal (EP): reflete a disposição individual e comportamental, que inclui emoções e características clássicas de personalidade - e.g., tipo A -que geram culpa, fracasso e ineficiência e comportamentos socialmente inadequados, tais como a evitação, a projeção e extrema reserva; e

8. Apoio pessoal (AP): reflete a disposição individual e comportamental, compatível com atitudes de autoconfiança, coping ou a habilidade para manejar e controlar eventos de vida,
Areias \& Guimarães satisfação da pessoa em "ser ela própria" e ter perspectivas congruentes com seus dados de realidade.

\section{ANÁLISE E PROCESSAMENTO DE DADOS}

Foram verificadas as seguintes hipóteses:

a) Existe interação entre Saúde Mental e fatores estressores e de apoio;

b) Os FPRs estão fortemente relacionados com os fatores estressores;

c) A Saúde Mental está fortemente associada com fatores de apoio;

d) Existe diferença significativa nos resultados para a SM e os FPRs de homens e de mulheres.

Procedeu-se às seguintes análises estatísticas: univariadas = Estatística Descritiva e Box - Plots; bivariadas $=$ Teste do Qui-quadrado com a finalidade de testar as hipóteses estatísticas; e multivariadas = Análise dos Componentes Principais e Correlação Linear de Pearson.

Para as análises uni e bivariadas foi utilizado o software STATISTICA 5.0, e para as análises multivariadas, o software WinSpad 3.5.

\section{RESULTADOS}

Relata-se a seguir o perfil demográfico e socioeconômico predominante dos trabalhadores pesquisados: sexo feminino (63\%), entre 30 a 49 anos (76\%), casados (49\%), categoria ocupacional de nível médio (47\%), escolaridade superior completa (36\%), com 0 a 3 dependentes (55\%), trabalho em tempo integral (80\%) com 40 horas de jornada (99\%), nível econômico médio $(67 \%)$, gastos médios por família/mês de 1 a 12 salários-mínimos (57\%) e rendimento médio família/mês de 7 a 18 saláriosmínimos $(52 \%)$.

Os dados da Tabela 1 indicam que os FPRs possuem correlação positiva com os fatores estressores e negativa com os fatores de apoio. Com a variável SM ocorre o contrário: há uma correlação negativa com os fatores estressores e positiva com os fatores de apoio. Existe interação entre saúde mental e fatores estressores e de apoio, e os FPRs estão fortemente relacionados com os fatores estressores e a saúde mental está fortemente associada com aos fatores de apoio. 
Gênero, estresse e trabalho

Tabela 1. Matriz de Correlação de Pearson para os 8 fatores

\begin{tabular}{lllllllll}
\hline & FPR & SM & ET & AT & ES & AS & EP & AP \\
\hline FPR & 1.00 & & & & & & & \\
SM & -0.58 & 1.00 & & & & & & \\
ET & 0.58 & -0.36 & 1.00 & & & & & \\
AT & -0.41 & 0.56 & -043 & 1.00 & & & & \\
ES & 0.66 & -0.43 & 0.68 & -0.34 & 1.00 & & & \\
AS & -0.59 & 0.75 & -0.44 & 0.59 & -0.49 & 1.00 & & \\
EP & 0.75 & -0.48 & 0.64 & -0.40 & 0.67 & -0.56 & 1.00 & \\
AP & -0.53 & 0.67 & -0.35 & 0.51 & -040 & 0,62 & -0.47 & 1.00 \\
\hline FPR - Fatores Psicossociais de Risco & ES - Estresse Social & \\
SM - Saúde Mental & & & AS - Apoio Social & \\
ET - Estresse no Trabalho & \multicolumn{7}{c}{ EP - Estresse Pessoal } \\
AT - Apoio no Trabalho & \multicolumn{7}{c}{ AP - Apoio Pessoal } \\
\hline
\end{tabular}

A seguir foi feita uma análise por componentes principais. Para essa análise consideraram-se como variáveis quantitativas principais FPR e SM; como variáveis quantitativas suplementares: ET, AT, ES, AS, $\mathrm{EP}$, e AP; e como variável categórica quantitativa suplementar o componente Gênero.

Utilizando apenas FPR e SM como variáveis principais, obteve-se o seguinte resultado: o primeiro componente explica 79\% dos dados (esse componente corresponde ao eixo horizontal do gráfico) e o segundo explica apenas $21 \%$ dos dados (eixo vertical). Então, nessa análise, será considerado o primeiro componente.

Observa-se na Figura 2 que as variáveis de apoio estão fortemente correlacionadas à $\mathrm{SM}$ e as variáveis estressoras estão fortemente correlacionadas aos FPRs.

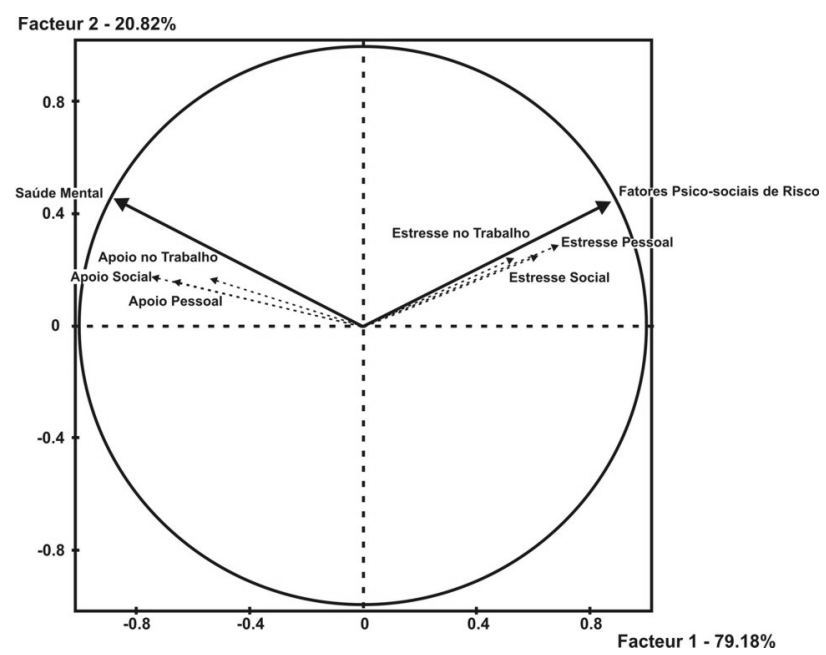

Figura 2. Correlação ente SM e apoios, fatores psicossociais de risco e estresse

A Tabela 2 apresenta a distribuição da variável gênero para as diferentes variáveis estudadas. Todas as médias para fatores psicossociais de risco e para os fatores de estresse (estresse no trabalho, estresse social e estresse pessoal) são maiores para o gênero feminino. As médias dos resultados para o fator saúde mental e para os fatores de apoio (apoio no trabalho, apoio social e apoio pessoal) são maiores para o gênero masculino do que para o feminino. Como a saúde mental tem correlação positiva com os fatores de apoio e os fatores psicossociais de risco têm uma correlação positiva com os fatores de estresse, fez-se análise univariada para as variáveis categóricas, porém só serão consideradas as variáveis quantitativas principais: FPR e SM.

Tabela 2. Distribuição dos Resultados segundo Gênero

\begin{tabular}{|c|c|c|c|c|c|c|c|c|c|}
\hline & GEN & FPR & SM & ET & AT & ES & AS & EP & $\mathbf{A P}$ \\
\hline Média & ASC & 4,78 & 21.27 & 9.12 & 18.72 & 4.97 & 19.27 & 5.52 & 17.48 \\
\hline DP & MASC & 3.29 & 3.47 & 4.15 & 3.79 & 3.60 & 3.70 & 3.49 & 2.55 \\
\hline Média & FEM & 6.17 & 19.52 & 9.46 & 18.36 & 5.17 & 17.98 & 6.06 & 16.90 \\
\hline DP & FEM & 3.84 & 4.05 & 4.02 & 4.36 & 3.72 & 4.31 & 3.45 & 3.18 \\
\hline \multicolumn{5}{|c|}{ DP - Desvio Padrão } & \multicolumn{5}{|c|}{ GEN - Gênero } \\
\hline \multicolumn{5}{|c|}{ MASC - Sexo Masculino } & \multicolumn{5}{|c|}{ FEM - Sexo Feminino } \\
\hline \multicolumn{5}{|c|}{ FPR - Fatores Psicossociais de Risco } & \multicolumn{5}{|c|}{ ES - Estresse Social } \\
\hline \multicolumn{5}{|c|}{ SM - Saúde Mental } & \multicolumn{5}{|c|}{ AS - Apoio Social } \\
\hline \multicolumn{5}{|c|}{ ET - Estresse no Trabalho } & \multicolumn{5}{|c|}{ EP - Estresse Pessoal } \\
\hline \multicolumn{5}{|c|}{ AT - Apoio no Trabalho } & \multicolumn{5}{|c|}{ AP - Apoio Pessoal } \\
\hline
\end{tabular}

A Figura 3 confirma a análise feita anteriormente: 0 gênero feminino apresenta um maior nível de FPR do que o masculino e um nível de SM menor. Também o box-plot para o gênero feminino apresenta maior dispersão dos dados tanto para FPRs como para SM.

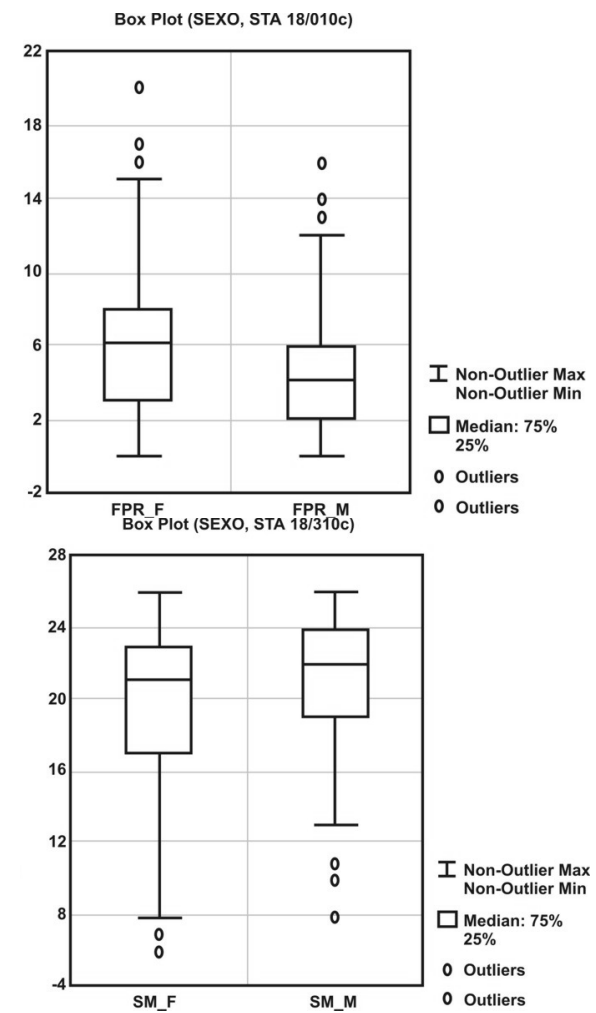

Figura 3. Distribuição de Fatores psicossociais de Risco e Saúde Mental por Gênero 
Foram feitos testes de hipóteses para cada fator estudado, segundo o gênero, os quais levaram aos seguintes resultados. (1) Há relação de dependência dos fatores psicossociais de risco em homens e mulheres, rejeitando-se $\mathrm{H}_{0}\left(X^{2}=12,08 ; \mathrm{p}=0,002\right)$, o que permite concluir que para o gênero masculino existem menores fatores psicossociais de risco do que para o feminino. (2) Existe relação de dependência entre saúde mental e a variável gênero, rejeitando-se $\mathrm{H}_{0}$ $\left(X^{2}=11,200 ; p=0,004\right)$, em que o gênero masculino apresenta maiores índices para a saúde mental do que o feminino. (3) Há relação entre estresse no trabalho e gêneros masculino e feminino, não se rejeitando $\mathrm{H}_{0}$ $\left(X^{2}=1,771 ; p=0,413\right)$, não se concluir pela relação de dependência entre as variáveis estudadas. (4) A relação de dependência entre apoio no trabalho e gênero não foi rejeitada $\mathrm{H}_{0}\left(X^{2}=1,178 ; p=0,555\right)$, e não há relação de dependência entre as variáveis estudadas. (5) Há relação de dependência entre estresse social e gênero, rejeitando-se $\mathrm{H}_{0} .\left(X^{2}=6,400 ; p=0,041\right)$ - em que se contata que homens têm menos estresse social do que mulheres. (6) Existe relação de dependência entre apoio social e gênero, não se rejeitando $\mathrm{H}_{0}$. $\left(X^{2}=5,515\right.$; $p=0,064)$, não havendo relações de dependência entre as variáveis estudadas. (7) Há relação de dependência entre $\mathrm{o}$ fator estresse pessoal e gênero, não se rejeitando $\mathrm{H}_{0 .}\left(X^{2}=1,292 ; p=0,524\right)$, e não se pode afirmar que haja relações de dependência entre as variáveis estudadas. (8) Existe relação de dependência entre apoio pessoal e gênero, não se rejeitando $\mathrm{H}_{0},\left(X^{2}=0,061 ; p=0,061\right)$, e não há dependência entre as variáveis estudadas.

\section{DISCUSSÃO}

Os dados obtidos mostram que os índices de saúde mental aumentam conforme aumentam os fatores de apoio e diminuem os fatores de estresse nas três dimensões: trabalho $(\mathrm{T})$, social $(\mathrm{S})$ e pessoal $(\mathrm{P})$, confirmando a primeira hipótese de estudo. Da mesma forma, os fatores psicossociais de risco aumentam conforme diminuem os fatores de apoio e aumentam os fatores de estresse nas três dimensões pesquisadas ( $\mathrm{T}$, $\mathrm{S}$ e P). Esses dados confirmam a segunda hipótese da pesquisa e corroboram a teoria proposta pelo modelo de estresse/apoio proposto por Ostermann (1989) utilizado, o qual postula que a saúde mental e os fatores psicossociais de risco são determinados tanto por apoios como por estressores nas dimensões - T, S e $\mathrm{P}$ - e que fatores de apoio podem anular os efeitos danosos do estresse.

Levi (1999) refere que alguns grupos apresentam maior risco para o estresse ocupacional e entre os fatores determinantes para esta ocorrência cita, entre outros, a combinação entre ser mulher e ter excesso de trabalho, uma situação econômica menos favorecida e um inadequado repertório de respostas para enfrentamento de determinadas situações.

Neste estudo, o gênero feminino apresenta mais fatores psicossociais de risco, estresse no trabalho, estresse social e estresse pessoal do que o masculino. Por outro lado, o gênero masculino apresenta melhores resultados em saúde mental e conseqüentemente em fatores de apoio (Trabalho, Social e Pessoal), confirmando a terceira hipótese deste estudo. De forma concordante com os achados acima expostos, Guimarães, Areias, e Caetano (1995; 1996), Guimarães, Areias, e Vieira (1996), Guimarães e Macfadden (1999), Areias, Guimarães, e Grubits (2000),em pesquisas anteriores com trabalhadores dessa mesma universidade, verificaram que os do gênero feminino apresentaram mais estressores externos do que os masculinos, portanto, estão mais expostos aos efeitos danosos do estresse.

Os fatores psicossociais de risco e o estresse social atingem mais os trabalhadores do gênero feminino do que os do masculino, e os índices de saúde mental são maiores para os do gênero masculino. Em pesquisa realizada por Mathews, Hertzman, Ostry, e Power (1998), mulheres relataram mais características negativas do trabalho que os homens, primeiramente devido à diferença de oportunidades de aprendizagem (26\% menos oportunidades comparadas com $13 \%$ de homens) e ao trabalho monótono (47 e $31 \%$ de homens). Mulheres empregadas em tempo integral relataram menos características negativas $(27 \%)$ do que as de período parcial (39\%) ou empregadas domésticas (36\%). Empregadas domésticas relataram menos oportunidades de aprendizagem $(36 \%)$ e maior monotonia $(49 \%)$ que os trabalhadores remunerados (21 e 22\% respectivamente), entretanto um menor número de empregadas domésticas relatou inabilidade quanto ao controle das etapas do trabalho (11\% comparadas com 23\%) e inflexibilidade quanto à possibilidade de poder efetuar intervalos ou paradas no trabalho segundo suas necessidades ( $21 \%$ comparadas com 47\%).

Uma vez confirmada a relação entre saúde mental e fatores de apoio e entre fatores psicossociais de risco e estresse nas três dimensões, pode-se concluir neste estudo que: os FPRs e as três dimensões do estresse $\mathrm{T}, \mathrm{S}$ e $\mathrm{P}$ - atingem mais os participantes do gênero feminino do que os do gênero masculino; por outro lado, apresentam maiores índices para a saúde mental e os fatores de apoio em todas as dimensões $-\mathrm{T}, \mathrm{S}$ e $\mathrm{P}-$, 
confirmando a quarta hipótese de estudo. Cabe lembrar que as mulheres, mesmo desempenhando um trabalho assalariado, como as deste estudo, são "incentivadas" a assumir primeiramente as responsabilidades da casa e da família, ficando então sujeitas a uma dupla sobrecarga de trabalho, especialmente quando os filhos ainda são pequenos. As mulheres, portanto, estão sujeitas aprioristicamente a um considerável dilema entre casamento e filhos e demandas ocupacionais. Kushnir e Kasan (1992) acrescentam que, de forma geral, as mulheres ocupam trabalhos hierarquicamente inferiores aos ocupados pelos homens, recebendo também menos benefícios e oportunidades de ascensão.

Fatores psicossociais de risco maiores em mulheres do que em homens podem indicar aumento dos sintomas e de queixas de doenças físicas associadas à instabilidade emocional, diminuição do tônus emocional e comportamento socialmente inapropriado, danoso e prejudicial. Grande número de mulheres vive numa cultura competitiva, ambiente que pode favorecer o aparecimento de sentimentos de inadequação e baixa auto-estima. Além disso, conflitos não resolvidos, autorepressão e expectativas podem produzir padrões de estresse e a ocorrência de doenças psicossomáticas com uma freqüência maior. Nesta direção, pesquisas epidemiológicas sobre o estresse ocupacional têm estudado a prevalência de transtornos mentais e físicos dos trabalhadores, o ambiente de trabalho, as demandas e recompensas no ambiente laboral, as categorias ocupacionais mais expostas aos riscos de adoecimento físico e/ou mental e o gênero como fator diferencial para resultados do nível de estresse experimentado (Miyata, Tanaka \& Tsuji, 1997; Stuart \& Halverson, 1997).

Neste estudo, o estresse pessoal e o estresse social também contribuem de forma negativa para as condições de vida das trabalhadoras. Esse fator pode refletir as condições de vida fora do âmbito de trabalho e inclui condições de vida caóticas, perigosas e insalubres, relações conflitivas na dinâmica familiar, no relacionamento com os amigos e vizinhos e responsabilidades aflitivas. As mulheres que concentram suas energias na carreira sentem-se freqüentemente culpadas ou preocupadas com o fato de ter deixado a família de lado. Além disso, o gasto de energia e dedicação para com marido e filhos pode desviar parte da energia que seria dirigida para a obtenção do sucesso profissional.

Alguns estudos mostram os que efeitos do trabalho remunerado sobre a saúde mental e o desempenho de múltiplos papéis têm tido mais benéficos do que adversos. Entretanto, a atitude negativa dos maridos em relação ao emprego remunerado de suas esposas pode resultar em conflitos conjugais e na falta de participação deles no cuidado dos filhos, anulando os efeitos potencialmente benéficos (Dennerstein, 1995).

\section{CONSIDERAÇÕES FINAIS}

A realização desta pesquisa, feita a partir de uma amostra composta de homens e mulheres pertencentes a estratos ocupacionais similares, diferentemente de outros estudos, possibilitou aferir diferenças significativas entre os mesmos, relativas às repercussões do estresse e apoios pessoal, do trabalho e social, bem como dos fatores psicossociais de risco na saúde mental.

Os dados obtidos neste estudo evidenciam que a saúde mental e os fatores de apoio estão interrelacionados, e que os fatores psicossociais de risco encontram-se relacionados ao estresse nas três dimensões estudadas: pessoal, do trabalho e social. Os FPRs e, conseqüentemente, o estresse nas três dimensões estudadas - P, T, S - mostram-se mais elevados para os participantes de gênero feminino. Os do gênero masculino apresentam maiores índices para a saúde mental e, de forma correspondente, para os fatores de apoio em todas as dimensões - P, T, S. Na amostra estudada, pessoas do gênero feminino apresentam mais estresse pessoal, social e no trabalho e mais fatores psicossociais de risco -ou seja, menores índices de saúde mental - do que o masculino, evidenciando maior risco para adoecimento físico e/ou mental.

A partir dos dados obtidos, outras pesquisas metodologicamente bem-conduzidas deverão ser feitas, direcionadas a diminuir os efeitos de outras variáveis confundidoras, para se ter maior visibilidade quanto à questão específica do gênero.

\section{REFERÊNCIAS}

Appelberg, K., Romanov, K., Heikkila, K., Honkasalo, M. L. \& Koshenvuo, M. (1996). Interpersonal conflict as a predictor of work disability: A follow-up study of 15.348 finnish employees. Journal of Psychosomatic Research, 40 (2), 157 167.

Areias, M. E. Q. (1999). Saúde mental, estresse e trabalho dos servidores de uma universidade. Tese de Doutorado NãoPublicada, Programa de Pós-Graduação em Saúde Mental da Faculdade de Ciências Médicas, Universidade Estadual de Campinas.

Areias, M. E. Q., Guimarães, L. A. M., \& Grubits, S. (2000). Stress and support in workers at a Brazilian university. In Books of abstracts. XXVII International Congress of Psychology (p. 78). Stockholm: Sweden. 
262

Artazcoz, L., Borrel, C. \& Benach, J. (2001). Gender inequalities in health among workers: the relationship with family demands. Epidemiology and Community Health, 55 (9), 639-647.

Barnett, R. C., Biener, L. \& Baruch, G. K. (1987). Gender and stress. Nueva York: Free Press.

Barnett, R.C., Marshall, N. L., Raudenbush, S. W. \& Brennan, R.T. (1993). Gender and the relationship between job experiences and psychological distress: a study of dual-earner couples. Journal of Personality and Social Psychology, 64(5), 794-806.

Barnett, R. C. (1998). Genero, estrés en el trabajo y enfermedad. Em Enciclopedia de Salud y Seguridad en el Trabajo (Vol. 2, pp. 339-348) Ginebra: Ministerio de Trabajo y Asuntos Sociales.

Cooper, C. L. \& Smith, M. J. (1985). Job stress and bluecollar work. Nueva York: Wiley.

Dennerstein, L. (1995). Mental health, work, and gender. International Journal of Health Services, 25(3), 503-509.

Goldberger, L. \& Breznitz, S. (1982). Handbook of stress. Nueva York: Free Press.

Guimarães, L. A. M., Areias, M. E. Q. \& Caetano, D. (1995). Risk of workers of developing physical or mental disorders due to external desirable or undesirable changes in life. [Abstract]. Em Societá Italiana de Psichiatria (Org.), XV World Congress of Social Psychiatry (p.118). Rome: Italy.

Guimarães, L. A. M., Areias, M. E. Q. \& Caetano, D. (1996). Stresful life events in university workers. [Abstract]. Em International Society of Psychiatry (Org.), X World Congress of Psychiatry, Book of Abstracts (p. 378). Madrid: Spain.

Guimarães, L. A. M., Areias, M. E. Q., \& Vieira, L. C. (1996). Changing society, work organization and mental health. [Abstract]. Em International Society of Psychiatry (Org.), X World Congress of Psychiatry, Book of Abstracts (p. 101). Madrid: Spain.

Guimarães, L. A. M. \& MacFadden, M. A. J. (1999). Validação para o Brasil do SWS ${ }^{\circledR}$ - Survey - Questionário sobre estresse, saúde mental e trabalho. Em L. A. M. Guimarães \& S. Grubits (Orgs.), Série Saúde Mental e Trabalho (Vol 1, pp.189-208). Casa do Psicólogo: São Paulo.

Holt, R. R. (1992). Occupational stress. Em L. Goldberger \& S. Breznitz (Org.), Handbook of stress (pp. 238-249). Nueva York: Free Press.

Kushnir, T. \& Kasan, R. (1992). Major sources of stress among women managers, clerical workers, and working single mothers: demands vs. resources. Public Health Review, 20(34), 215-229.
Areias \& Guimarães

Levi, L. (1999) Guía sobre el estrés relacionado con el trabajo: La "sal de la vida "o el "beso de la muerte?". Em Dirección General de Empleo y Asuntos Sociales de la Comisión Europea de Seguridad y salud en el trabajo (Org.). Barcelona.

Ludermir, A. B. (2000). Inserção produtiva, gênero e saúde mental. Cadernos de Saúde Pública, 16(3), 123-129.

Marques, M. B. (Org.). (1996). Declaração de Helsinque. Em Search of a national forum for bioethics in Brazilian public policy. Cadernos Saúde Pública, 12(4), 443-454.

Mathews, S., Hertzman, C., Ostry, A. \& Power, C. (1998). Gender, work roles and psychosocial work characteristics as determinants of health. Social Science \& Medicine, 46(11), 1417-1424.

Miyata, M., Tanaka, Y. \& Tsuji, S. (1997). Occupational stress as the cause of psychosomatic and mental disorders. Sangyo Ika Daigaku Zasshi, 19(4), 297-305.

Ostermann, R. F. (1989). The SWS stress/support model: School of Psychology: Farleigh Dickinson University, Paramus, New York: Free Press.

Ostermann, R. F., Gutierrez, R. E. (1992). The SWS- Survey: cross cultural assessment of positive/negative mental health and stress variables. [Resumo]. Em International Society of Psychology (Org.), XII International Congress of Psychology (p.236). Brussels: Belgium.

Ostermann, R. F., Gutiérrez, R. E., Contreras, C. \& Atenco, F. (1995). Encuesta SWS ${ }^{\odot}$ - survey de estrés, salud mental y trabajo. [Resumo]. Em Sociedad Mexicana de Psicología (Org), VII Congreso Mexicano de Psicología, Book of Abstracts (p.123). Guadalajara.

Santana, V. S., Loomis, D. P., \& Newman, B. (2001). Housework, paid work and psychiatric symptoms. Revista de Saúde Pública, 35(1), 145- 150.

Sauter, S. L., Hurrell Jr, J. J., \& Cooper, C. L. (1989). Job control and worker health. Chichester: John Wiley \& Sons.

Stuart, J. A., \& Halverson, R. R., (1997). The psychological status of U.S. Army soldiers during recent military operations. Military Medicine, 162(11), 737-743.

Simon, R. W. (1995). Gender, multiple roles, role meaning, and mental health. Journal of Health Society Behavior, 36(2), 182-194.

Recebido em 02/02/2004 Aceito em 07/07/2004

Endereço para correspondência: Maria Elenice Quelho Areias. Rua Fernão Lopes, 544, Parque Taquaral, CEP 13087-050, Campinas-São Paulo. E-mail: elenice@ cecom.unicamp.br 